\title{
John Mason Clarke e os lingulídeos e discinídeos dos estratos devonianos da Bacia do Paraná, estado do Paraná, Brasil: estado da arte
}

\author{
John Mason Clarke and the devonian lingulids and discinids of \\ the Paraná Basin, Parana state, Brazil: work of art
}

\author{
John Mason Clarke y lingulideos y discinideos y estratos del \\ Devónico de la Cuenca del Paraná, estado del Paraná, Brasil: \\ estado del arte.
}

\author{
Carolina Zabini \\ cazabini@gmail.com \\ Universidade Federal do Rio Grande do Sul \\ Jeanninny Carla Comniskey \\ jcomniskey@usp.br \\ Universidade de São Paulo \\ Elvio Pinto Bosetti \\ elvio.bosetti@pq.cnpq.br \\ Universidade Estadual de Ponta Grossa
}

Resumo: John Mason Clarke descreveu as espécies de lingulídeos e discinídeos ocorrentes nos estratos devonianos da Bacia do Paraná, e as comparou com outras espécies ocorrentes no atual hemisfério norte. Clarke (1913) propôs duas novas espécies de discinídeos e quatro de lingulídeos infaunais. $O$ presente trabalho resgata a memória de Clarke e retrata o estado da arte dos lingulídeos do devoniano paranaense. Atualmente reconhecem-se, além de Orbiculoidea sp., dois novos gêneros de discinídeos (Rugadscina sp. e Gigadiscina sp.) ocorrentes nos estratos devonianos. Já a sistemática dos lingulídeos infaunais permanece em aberto, uma vez que o gênero Lingula não é mais considerado paleozoico. Tanto discinídeos quanto lingulídeos infaunais apresentam assinaturas tafonômicas que retratam relações ecológicas com outros grupos animais. Discinídeos são, por vezes, encontrados incrustrados por briozoários e lingulídeos podem apresentar perfurações em sua carapaça. Em relação à tafonomia, os discinídeos podem ser encontrados isolados (com valvas inteiras articuladas, e inteiros desarticulados e/ ou ainda fragmentados), representando ambientes de shoreface e offshore, ou agrupados em ambientes de shoreface proximal a distal. Já os lingulídeos infaunais ocorrem em posição de vida principalmente em ambientes de foreshore, sendo que o grau de articulação diminui ao longo do perfil batimétrico (mais para o fundo) e a fragmentação aumenta ao longo do referido perfil.

Palavras-chave: Lingulida. Brachiopoda. Formação Ponta Grossa. Formação São Domingos. Sistemática. Tafonomia 
Abstract: John Mason Clarke described devonian lingulids and discinids species occurring at the Paraná Basin, Brazil, and compared them to other species of the northern hemisphere. Clarke (1913) proposed two new discinid species, and four new lingulid species. The present paper recovers Clarke's work and presents the work of art of these Devonian brachiopods. Nowadays two new discinid genera are recognized for these strata (Rugadscina sp. e Gigadiscina sp.) and the already known Orbiculoidea sp. remain. The lingulid taxonomy is still in study and remains opened, once Lingula is not considered a Paleozoic genus anymore. Discinids and lingulids show taphonomic signatures that reflect past ecological interactions. Discinids are found incrusted with bryozoans, and lingulids can occur with circular perforations. In relation to their taphonomy, discinids can be found isolated (as complete and articulated valves, or as complete and disarticulated valves and/or fragmented valves), representing shoreface and offshore environments. Discinid grouped valves occur in proximal to distal environments. Lingulids can be found in life position at the foreshore. The degree of articulation decreases with increasing paleobathymetry and the inverse relation occurs with the fragmentation degree, wich increases with increasing paleobathymetry.

Keywords: Lingulids. Discinids. Ponta Grossa Formation. São Domingos Formation. Taxonomy. Taphonomy.

Resumen: John Mason Clarke describió especies presentes en discinideos lingulídeos y estratos del Devónico de la cuenca del Paraná, y en comparación con otras especies en el hemisferio norte en la actualidad. Clarke (1913) propuso dos nuevas especies y cuatro discinídeos lingulídeos infaunal. En este trabajo se recuerda la memoria de Clarke y retrata el estado de la técnica lingulídeos Devónico Paraná. Actualmente se reconocen, además Orbiculoidea sp., Dos nuevos géneros de discinídeos (Rugadscina sp . Gigadiscina y sp.) Que ocurren en el Devónico estratos. Ya sistemática lingulídeos infaunal permanece abierta, ya que el género Lingula ya no se considera paleozoico. Ambos discinídeos como lingulídeos infaunales presentes firmas tafonómicos que representan las relaciones ecológicas con otros grupos de animales. Discinídeos se encuentran a veces con incrustaciones de briozoos y lingulídeos puede tener agujeros en su caparazón. En cuanto a la tafonomía los discinídeos se encuentran aisladas (con válvulas enteras articulados y toda desarticuladas y/o fragmentada ) que representa frente de playa y ambientes marinos o en entornos agrupados shoreface proximal a distal. Lingulídeos infaunal ya se produce en la posición de la vida de la zona de mareas especialmente en ambientes, donde el grado de articulación disminuye con el perfil batimétrica (más cerca de la parte inferior) y los aumentos de fragmentación a lo largo de dicho perfil.

Palabras clave: Lingulida. Braquiópodos. Ponta Grossa Formación. Formación de São Domingos. Sistemática. Tafonomía. 


\section{A ORDEM LINGULIDA E SUA OCORRÊNCIA NOS ESTRATOS DEVONIA- NOS DA BACIA DO PARANÁ}

Os representantes da ordem Lingulida são braquiópodes infaunais filtradores que possuem o corpo protegido por uma concha formada por duas valvas quitinofosfáticas; a articulação destas valvas se dá por um complexo arranjo muscular. O pedículo emerge entre as valvas ou através da valva pedicular, e é a estrutura responsável pela fixação nos substratos. O formato da concha pode variar desde alongada oval, circular a subtriangular. Nos discinídeos as duas valvas podem ser cônicas, entretanto é mais comum encontrar a valva dorsal cônica e a valva pedicular plana, já nos lingulídeos infaunais, ambas as valvas são semelhantes em formato e tamanho e ambas são convexas. A ordem Lingulida apresenta distribuição estratigráfica a partir do Cambriano até o Holoceno. Os lingulídeos foram primeiramente identificados na Bacia do Paraná pela Comissão Geológica do Império do Brasil (1876-1877) (ZABINI et al., 2007; COMNISKEY, 2011).

Clarke (1913) e Kozlowski (1913) foram os primeiros a descrever o grupo para o Devoniano do estado do Paraná. Clarke (1913) reconheceu a espécie Orbiculoidea baini Sharpe (1856), e propôs duas espécies novas: O. bodenbenderi e O. collis (Fig. 1). Kozlowski (1913) também descreveu uma nova espécie de discinídeo (Orbiculoidea grandissima) que apresentava as mesmas características que $O$. collis de Clarke. A prioridade de termos foi conferida a Clarke devido ao mês de publicação de seu trabalho, anterior ao de Kozlowski (março e novembro de 1913, respectivamente). Em 1943, Frederico Waldemar Lange propôs uma nova espécie, Orbiculoidea excentrica. Após estes trabalhos, por aproximadamente 60 anos os discinideos ficaram sem estudos mais aprofundados no devoniano brasileiro. Retomam os trabalhos Comniskey \& Bosetti (2011, 2012), e Comniskey (2011), que abordam novas questões quanto à sistemática, bioestratigrafia, paleoecologia e tafonomia do grupo para as camadas do Devoniano da Bacia do Paraná.

Comniskey \& Bosetti (2011) demonstram um grande diferencial de Orbiculoidea collis em relação aos outros do mesmo gênero. Mergl e Massa (2005) propuseram um novo gênero para a Bacia de Tamesna, Sul da Argélia, e sinonimizaram O. collis Clarke, 1913, ocorrente nesta bacia para o novo gênero, Gigadiscina, de grandes discinídeos. Após analisar as amostras encontradas na Formação Ponta Grossa, nota-se uma grande similaridade com a descrição da Gigadiscina collis, por apresentar um tamanho superior do que as Orbiculoidea encontradas, linhas concêntricas finas e uma fenda pedicular fina. Após discussões e observações sobre os dois gêneros, os autores decidiram pela mudança de gênero da espécie Orbiculoidea collis para Gigadiscina collis, pelas justificativas apresentadas por Mergl e Massa (2005) em seu trabalho. 
Figura 1. Espécies de discinídeos descritas e estampadas em Clarke (1913): A-D -Orbiculoidea collis; E-F - Orbiculoidea baini; G-J - Orbiculoidea bodenbenderi.(modificado de Clarke, 1913)
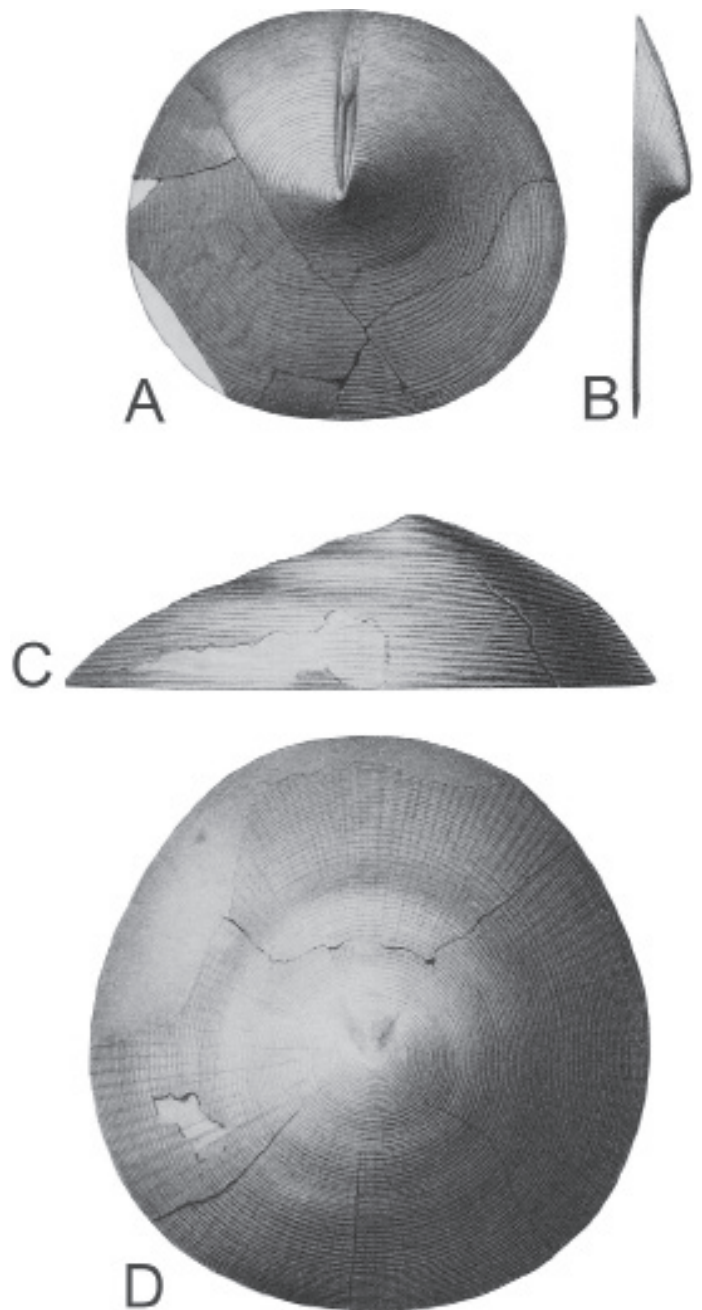
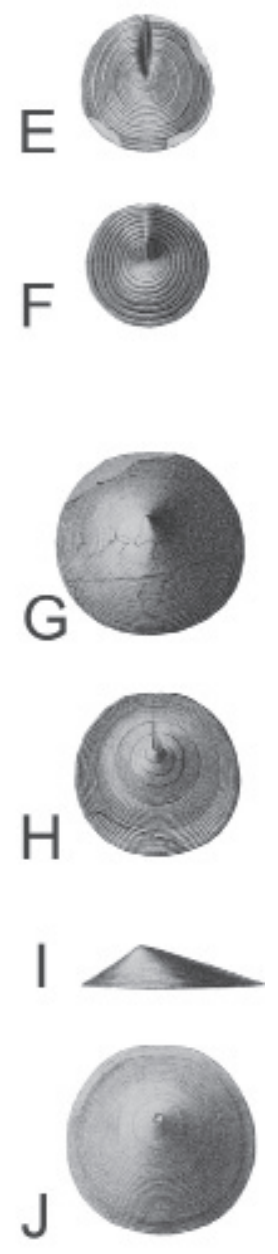

Comniskey \& Bosetti (2012) propõem a ocorrência de um novo gênero (Rugadiscina) para o Devoniano da Bacia do Paraná. Este novo gênero foi proposto por Mergl (2006) para as camadas Silurianas da Inglaterra.

Para a outra super-família Clarke (1913) registrou também cinco espécies de Lingula, quatro das quais inéditas para a época: Lingula scalprum Clarke, Lingula lepta Clarke, Lingula keideli, Clarke, Lingula lamella, Clarke e Lingula subpunctata Knod (Fig. 2). No entanto, as diferenças entre estas espécies eram propostas com base na forma geral da concha. Foi somente com o trabalho de Bosetti (1989) que o arranjo dos músculos e dos contornos das cicatrizes musculares, a morfologia da região umbonal e a disposição dos canais anteriores do manto foram utilizadas na proposição de duas espécies: Lingula lilliata e Lingula sagittalis. Já Emig (2003) reduziu a distribuição temporal da Família Lingulidae (a família teria aparecido somente no início do Mesozoico e o gênero (Lingula) ocorreria no final do Cretáceo, início do Terciário (ZABINI et al., 2007; ZABINI 2007, 2011). A té o momento os exemplares de lingulídeos infaunais dos estratos devonianos do Paraná não foram classificados em outro gênero. 
Figura 2. Espécies de lingulídeos infaunais descritas e estampadas em Clarke (1913): A - Lingula scalprum; B - Lingula lepta; C - Lingula keideli; D - Lingula subpunctata; E - Lingula lamella. (modificado de Clarke, 1913)
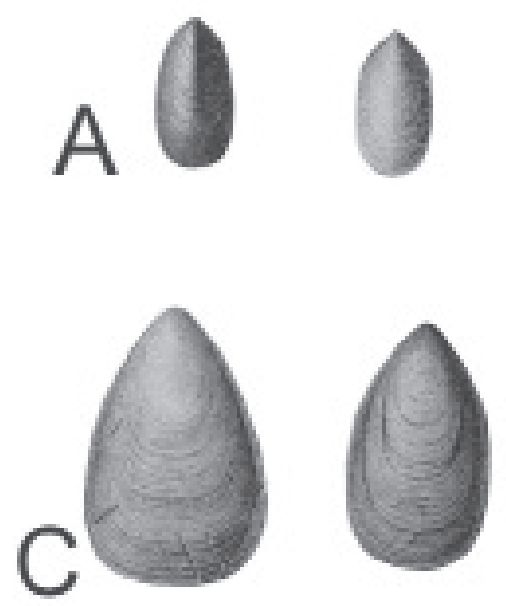

\section{MATERIAL E MÉTODOS}

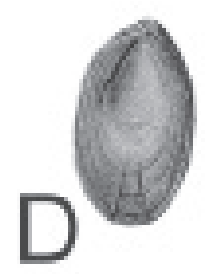

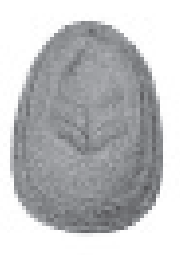

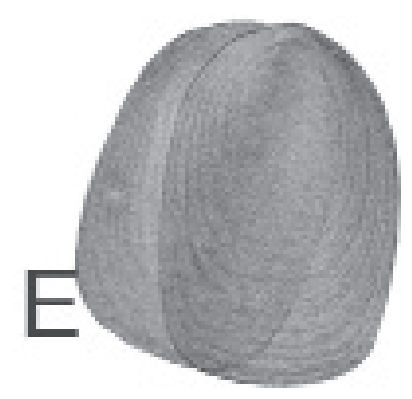

O presente trabalho representa o estado da arte do conhecimento da Ordem Lingulida para o Devoniano do Estado do Paraná, Bacia do Paraná, Brasil. Ele é o resultado de dois projetos desenvolvidos com a referida ordem, na última década. Estes projetos foram publicados de forma independente em Comniskey (2011) e Zabini (2007 e 2011), e são aqui apresentados de forma conjunta, como o estado da arte do conhecimento desta Ordem (Lingulida), desde os tempos de Clarke até a atualidade.

Comniskey (2011) analisou 1.499 amostras com as numerações de DEGEO/MPI: 5000 até 6499 . Zabini (2011) analisou 2.700 amostras contendo cerca de 8.000 bioclastos. $\mathrm{O}$ material está depositado no Laboratório de Estratigrafia e Paleontologia da Universidade Estadual de Ponta Grossa, com as seguintes numerações: DEGEO/MPI: 0001 a 0599, 1839 a 1902,2535 a 2659 e 3000 a 4587.

O detalhamento das técnicas de coleta e análise podem ser encontradas nos trabalhos originais de Comniskey (2011) e Zabini (2007, 2011).

\section{GEOLOGIA DA ÁREA}

A Bacia do Paraná possui cerca de $1.600 .000 \mathrm{~km}^{2}$ localizada no sul, centro-oeste e sudeste do Brasil, norte/noroeste do Uruguai, parte do Paraguai e Argentina. Os estados de São Paulo, Paraná, Santa Catarina (regiões nordeste, central e ocidental) e Rio Grande do Sul (central e ocidental) e parte dos estados de Mato Grosso, Mato grosso do Sul e Goiás (regiões norte e noroeste) estão localizados nesta bacia (PETRI \& FÚLFARO, 1983; SCHNEIDER et al., 1974). No estado do Paraná afloram, entre outros, depósitos sedimentares marinhos do Período Devoniano.

Os estratos devonianos serão aqui representados de acordo com a proposta de Grahn et al. (2013), no qual: a sequência A de Bergamaschi (1999) é Lochkoviana; a 
sequência B é Neopraguiana a Eoemsiana; a porção basal da sequência C é Neoemsiana final; as sequências C-F abrangem o Neoemsiano final ao Eogivetiano; e a sequência F está presente somente em subsuperfície (NeoGivetiano-Neofrasniano inicial)(Fig. 3).

Os trabalhos com lingulídeos (Ordem: Lingulida) baseiam-se em prospecções de campo realizadas essencialmente nas sequências B a D da sub-bacia de Apucarana, Bacia do Paraná, Estado do Paraná, Brasil (Fig. 4). Maiores informações sobre os pontos de coleta podem ser encontrados em Comniskey (2011) e Zabini $(2007,2011)$.

Figura 3. Comparação entre a classificação litoestratigráfica e as sequências de Grahn et al. (2013) para os estratos devonianos da Bacia do Paraná, no Paraná.

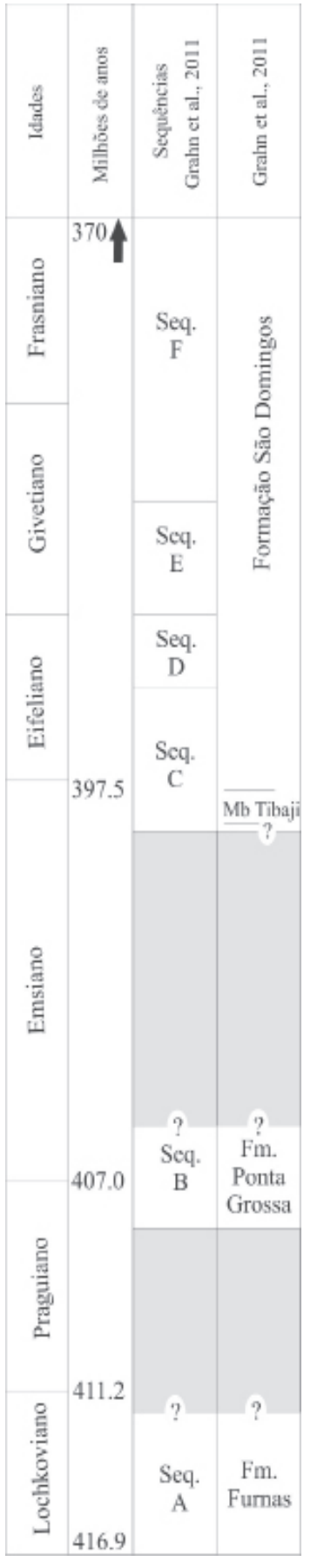


Figura 4. Mapa mostrando os locais de coleta e a ocorrência de rochas devonianas no estado do Paraná.
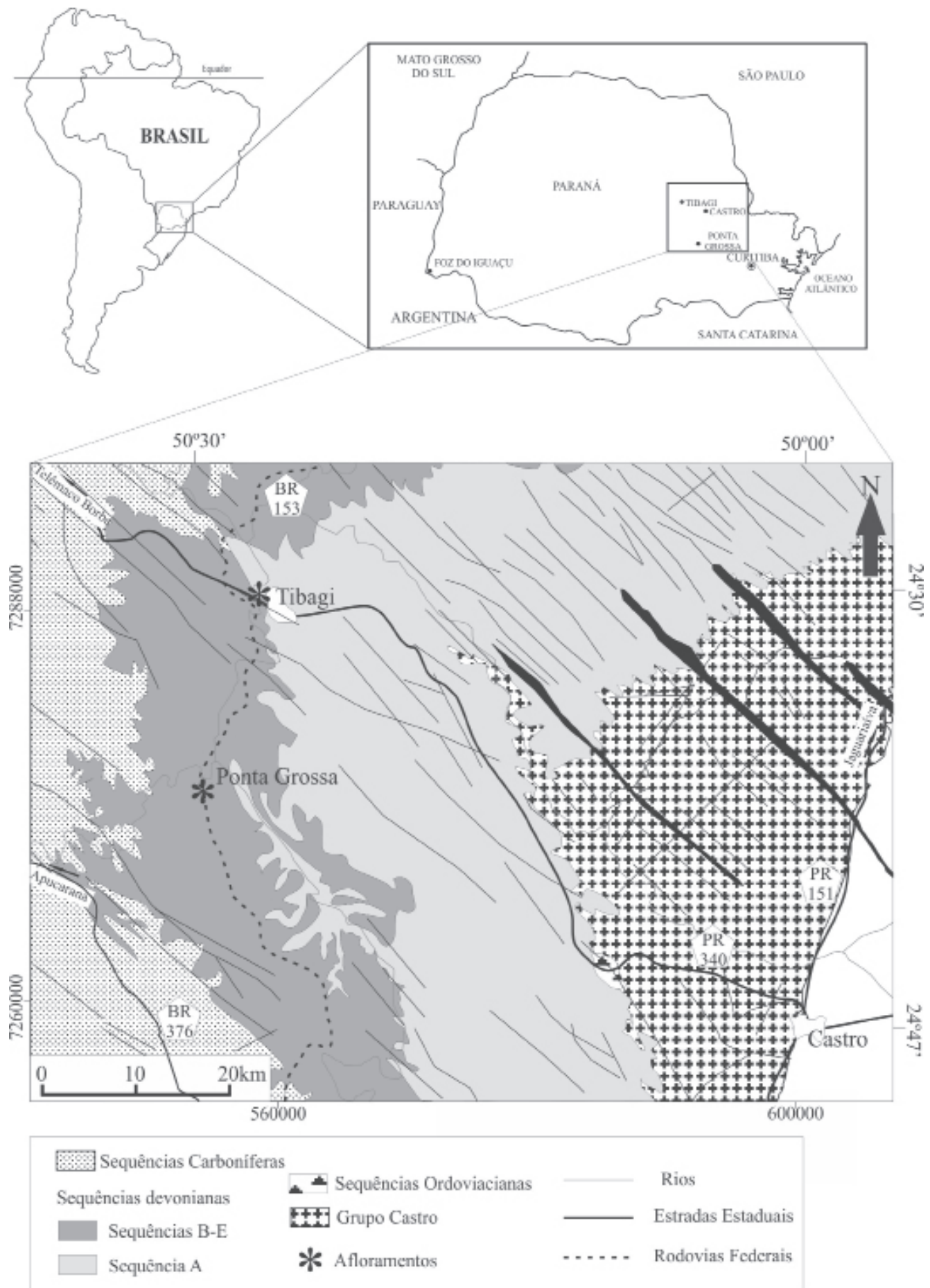

\section{PALEOECOLOGIA DISCINIDEOS}

Discinideos são braquiópodes inarticulados de hábito exclusivamente marinho. Eles são encontrados desde a zona litoral até a zona batial (WILLIANS \& ROWEL, 1997). O registro sobre sua biogeografia e ecologia é escasso (EMIG, 1997). Segundo Mergl (2001), ambientes de recife onde a energia do ambiente é alta, são pobres em lingulídeos, 
entretanto, em ambientes pouco mais profundos e com menos energia, eles são facilmente encontrados.

O hábito de vida dos discinídeos fósseis foi discutido por muito tempo, porém trabalhos mais recentes (SÜDKAMP, 1997; MERGL, 2001; MERGL \& MASSA, 2005; MERGL, 2010; COMNISKEY, 2011) afirmam um hábito epibentônico séssil, para o grupo. Os discinídeos são fixados ao substrato ou até mesmo em outros animais através do pedículo, apesar de este ser encurtado em algumas espécies; o pedículo possui alta flexibilidade facilitando a movimentação do animal. Algumas feições específicas, tais como valvas pediculares mais planas sugerem uma fixação mais firme ao substrato, mantendo, ao mesmo tempo, uma boa flexibilidade para a movimentação do pedículo (MERGL, 2001).

De acordo com Mergl (2010), discinídeos - tanto espécies viventes quanto as extintas - não possuem preferência quanto ao hábito de vida isolado ou agregado. LaBarbera (1985), em seu estudo com Discradisca strigata, observou que estes animais formavam assembleias capazes de prevenir ou reprimir o crescimento de invertebrados competidores através da abrasão e desgaste da epifauna e o esgotamento dos nutrientes na água. Mergl (2010), afirma que esse tipo de comportamento gregário indica que os discinideos extintos tinham a mesma ecologia e o mesmo pedículo discoidal, adaptado a substratos duros, como o de algumas espécies viventes (e.g. Discinisca lamellosa). O autor sugere que estes agrupamentos diminuiriam o estresse da predação ou ainda, tal qual acontece com alguns moluscos gastrópodes, facilitariam a fertilização pela proximidade dos indivíduos. Este padrão gregário foi observado entre os discinídeos do Devoniano da Bacia do Paraná, em que valvas encontram-se sobrepostas umas às outras, formando verdadeiras assembleias de discinideos.

\section{RELAÇÕES PRETÉRITAS COM OUTROS GRUPOS}

Amostras de Orbiculoidea baini do neo-Emsiano da Bacia do Paraná apresentaram incrustação de briozoários. Os zoécios tem formato prismático, por vezes alongado, e ocorreram incrustados sobre as porções marginais das valvas pediculares.

Segundo LaBarbera (1985), braquiópodes inarticulados da epifauna que habitavam substratos rígidos, competiam por espaço com outros animais da epifauna, desde o final do Cambriano. Animais coloniais (esponjas e briozoários) são competidores superiores se comparados aos inarticulados solitários como os discinideos, e possuem uma preferência em se alojar nas margens da valva pedicular destes animais. Para LaBarbera (1985) as esponjas e briozoários possuem certa predileção em habitar as valvas pediculares dos discinídeos, entretanto, os discinideos têm se aperfeiçoado em técnicas eficazes contra estes competidores. A presença do pedículo e sua mobilidade acabam com as chances dos animais "invasores" crescerem em torno do braquiópode, portanto, as espécies de discinídeos com pedículo central estão mais protegidas contra o ataque destes animais.

\section{LINGULÍDEOS INFAUNAIS}


Lingulídeos são animais quitinofosfáticos de hábito de vida infaunal e que se prendem ao fundo de seu local de vida (terrier) através do pedículo.

Lingulídeos infaunais atuais vivem em sedimentos compactados e estáveis sob a influência de correntes de fundo moderadas (PAINE, 1970; EMIG, 1984). Seus substratos preferenciais são aqueles formados por areia fina a muito fina, além de areia argilosa e areia grossa, em uma matriz arenosa fina a muito fina. A salinidade preferencial desses lingulídeos atuais é normal, apesar de responderem bem ao estresse osmótico, por curtos períodos de tempo (EMIG, 1997). Além disso, lingulídeos infaunais atuais mostram uma boa resposta a ambientes pouco oxigenados (YATSU, 1902; WORCESTER, 1969), em comparação a moluscos bivalves, por exemplo (EMIG, 1997).

Os lingulídeos infaunais fósseis dos estratos devonianos da Bacia do Paraná ocorrem em ambiente marinho, em posição de vida (PV), ao longo de todo o perfil batimétrico, tendo, no entanto, preferência pelas porções de foreshore (em geral, formado por areia fina a média). Lingulídeos infaunais em PV ocorrem em pequenas quantidades ao longo do shoreface e do offshore transicional, e tornam a ocorrer em abundância no offshore, onde, por vezes, têm seu potencial de preservação aumentado. Lingulídeos infaunais desarticulados, fragmentados e concordantes ao plano de acamamento são encontrados no shoreface distal e ao longo do offshore transicional (ZABINI \& BOSETTI, 2011).

De acordo com os dados obtidos dos fósseis, e em comparação com os dados dos seus pares recentes, é possível afirmar que o hábito de vida desses lingulídeos, e suas estratégias de sobrevivência, permaneceram as mesmas ao longo de todo o Fanerozóico.

\section{RELAÇÕES PRETÉRITAS COM OUTROS GRUPOS}

Algumas valvas de lingulídeos infaunais dos estratos devonianos da Bacia do Paraná apresentam perfurações circulares (ZABINI et al., 2006a, b), e as estampas de Clarke (1913) mostram exemplares de lingulídeos com perfurações tubulares (Fig. 2C).

Em relação às perfurações circulares, sua natureza predatória ou parasítica não pode ser confirmada. No entanto o fato de que alguns exemplares estudados apresentam uma variedade de perfurações de diferentes tamanhos e em diferentes posições na concha, são fortes indícios de relações de parasitismo (Fig. 5). Pesquisas realizadas com fósseis Paleozóicos (BAUMILLER et al., 1999; WILSON \& PALMER, 2001; HOFFMEISTER et al., 2004) sempre consideram tais estruturas como relações predação/parasitismo, uma vez que o organismo perfurador normalmente não é conhecido. Kowalewski et al. (1997) destaca que a predação em lingulídeos atuais é ocasional e rara, e como nos exemplares paleozoicos estudados, a maioria das valvas com esta assinatura apresenta diversas perfurações. Já as perfurações tubulares, como observadas e desenhadas por Clarke (1913) não foram observadas no material analisado no presente trabalho. 
Figura 5. Valva de lingulídeo infaunal com perfuração circular em sua porção central-posterior. Escala de $1 \mathrm{~cm}$.

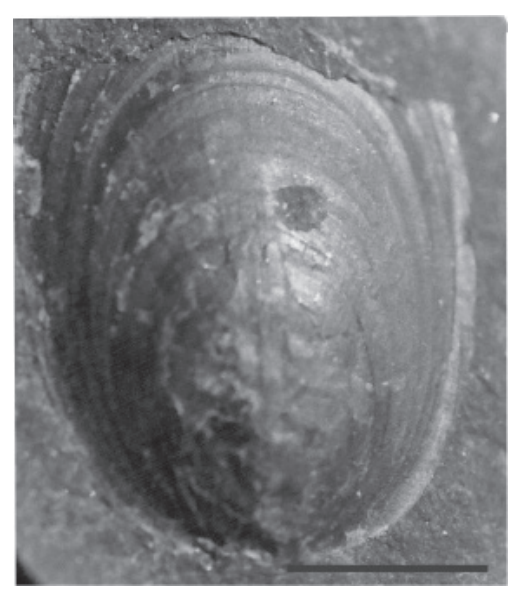

\section{PADRÕES TAFONÔMICOS DISCINÍDEOS}

Os discinídeos são comuns nos arenitos médios de shoreface e nos siltitos do offshore transicional. Duas classes tafonômicas foram determinadas para os discinídeos do Devoniano da Bacia do Paraná, são elas: agrupamentos e isolados.

A classe de discinídeos agregados é encontrada em ambientes de shoreface distal à proximal, podendo ser divididos em três subclasses: A1) indivíduos com valvas inteiras articuladas próximas ou sobrepostas; A2) associação de valvas pediculares e braquiais inteiras, desarticuladas, próximas ou sobrepostas, e A3) fragmentos de valvas associadas a valvas inteiras articuladas ou desarticuladas.

Os discinídeos isolados podem ser divididos em duas subclasses: B1) indivíduos com valvas inteiras articuladas, e B2) indivíduos inteiros desarticulados e/ ou fragmentados. Esta classe ocorre em ambientes de shoreface e offshore (Fig. 6).

As classes distribuem-se conforme a paleobatimetria inferida a partir da litologia onde o material foi coletado (WALKER \& PLINT, 1992). Gigadiscina collis, Orbiculoidea bodenbenderi e Rugadiscina sp. tem preferência por ambientes mais rasos, enquanto O. baini e O.excentrica são registrados tanto em ambientes rasos como em ambientes mais profundos. Em geral, a fauna da Província Malvinocáfrica, quando ocorrente em ambientes de offshore, sofreu algum tipo de transporte, e indicam uma tentativa de re-estabelecimento em ambientes não preferenciais (ZABINI et al, 2010; ZABINI \& BOSETTI, 2011). Este parece ser o caso das duas últimas espécies citadas, que podem ter sido removidas para águas mais profundas via fluxo energético de maior intensidade, e tentariam o re-estabelecimento pela nova fixação ao substrato. Isto explicaria o fato de que nos folhelhos pretos de offshore os fósseis comumente são encontrados isolados. 
Figura 6. Modelo geral que ilustra a ocorrência de discinídeos e lingulídeos infaunais e de suas classes tafonômicas ao longo de um perfil batimétrico. Ver texto para maiores detalhes.

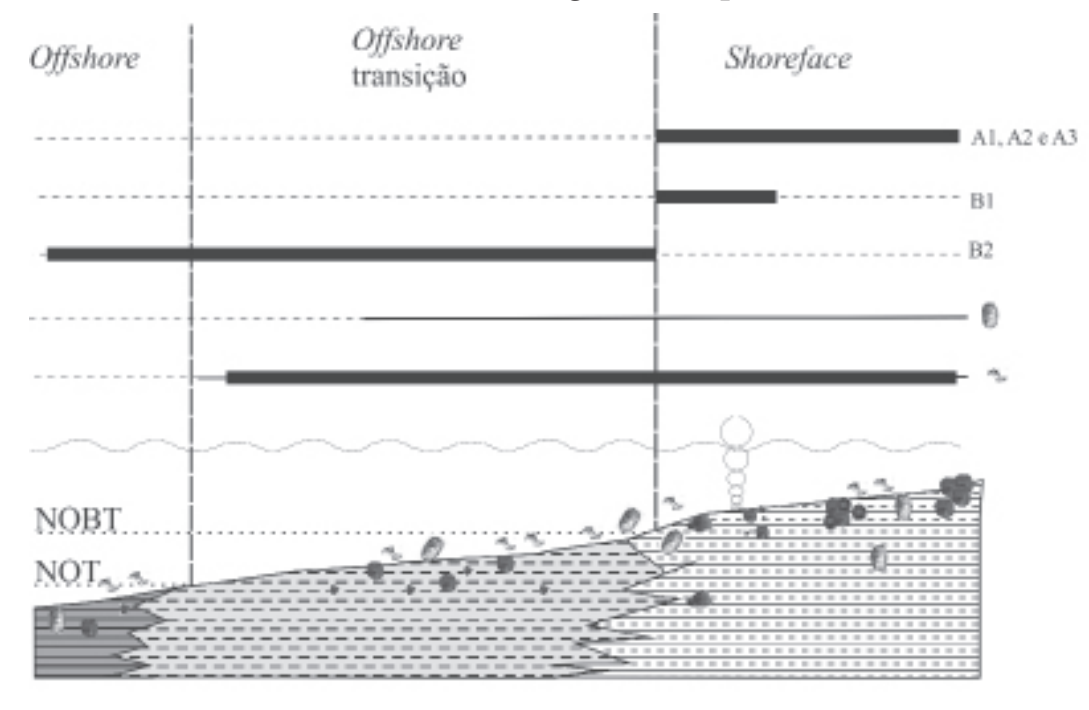

\section{LINGULÍDEOS INFAUNAIS}

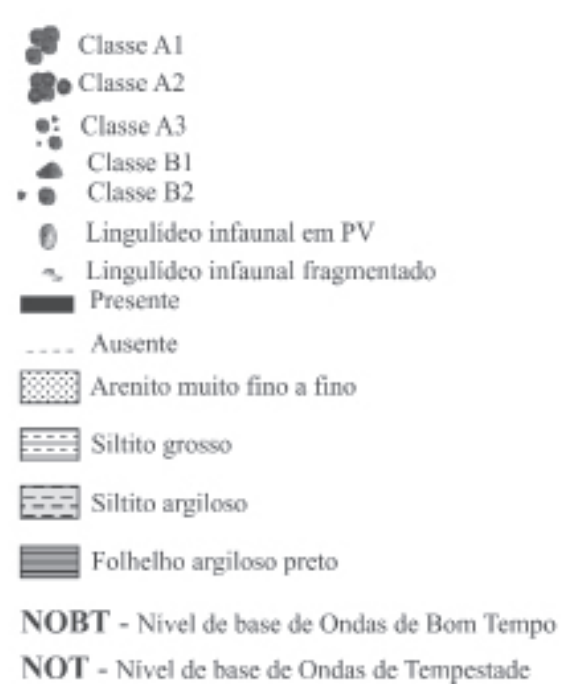

De acordo com Zabini et al. (2012) é possível identificar três modos de ocorrência de lingulídeos infaunais em relação ao plano de acamamento nos estratos devonianos da bacia do Paraná: a) perpendiculares com valvas pareadas (posição de vida), b) oblíquos, valvas articuladas e paralelas ao plano, e c) valvas desarticuladas inteiras ou em vários graus de fragmentação.

De acordo com estes modos de ocorrência, duas tafofácies principais foram propostas:

Tafofácies 1 (TF1) representa valvas de lingulídeos desarticuladas, inteiras ou fragmentadas, ocorrendo da parte distal do shoreface e, principalmente, ao longo do offshore transicional. Não há seleção de valvas por tamanho neste gradiente, porém o que se verifica é que os tamanhos dos fragmentos de valvas de lingulídeos são menores na porção distal do offshore transicional (logo acima ou abaixo do nível de base de ondas de tempestade). Valvas de lingulídeos orientados na forma de cordas e rosetas são também encontrados preferencialmente na porção distal do offshore transicional, explicada pela ação de correntes de turbidez formadas por lama e areia fina. A movimentação desta corrente vai inicialmente perdendo sua porção de areia e permanecendo com a lama e com isso os sedimentos finos vão formando uma pluma mais leve e que flutua. Parte da corrente sobe, num processo chamado de flutação reversa (reversal bouyancy) (STOW \& WETZEL, 1990; SPARKS et al., 1993; PRITCHARD \& GLADSTONE, 2009) e vai perdendo energia até parar; sugere-se que este processo forneça energia suficiente capaz de reorientar as valvas que ali se encontram. Além de valvas concordantes ao plano de acamamento, também são encontradas, em menor número, valvas perpendiculares ao sedimento.

Tafofácies 2 (TF2), nesta tafofácie é onde o maior número de valvas em posição de vida são descritas. Esta tafofácies é subdividida em duas: 
Tf2a - com lingulídeos infaunais em posição de vida, preservados como moldes ou impressões, em porções do foreshore ou do shoreface;

Tf2b - lingulídeos infaunais em posição de vida preservados em concreções, associados a valvas desarticuladas, em porções do offshore, abaixo do nível de base de ondas de tempestade. Estas duas tafofácies fornecem uma visão diferenciada daquela fornecida pela literatura mais antiga (ANELLI et al., 1997; NUNES, 1999; SIMÕES et al., 1999a, b) (Fig. 6).

A presença de fragmentos de valvas de lingulídeos infaunais no registro da sucessão devoniana estudada era negligenciada. Os estudos tafonômicos prévios a Zabini (2007) relatavam tão somente a presença de patches de lingulídeos infaunais, provavelmente influenciados por poucos dados de campo e pelos dados atualistas que afirmavam serem raras as preservações de fragmentos de lingulídeos. Pequeno número de valvas em posição de vida estão quase sempre associados à grande número de valvas concordantes ao plano de acamamento; talvez estas valvas em posição de vida possam representar lingulídeos infaunais que chegaram vivos após algum transporte por corrente e que reescavaram o substrato para produzir um novo local de vida, conforme discutido também por Zonneveld \& Greene (2010). Isso porque o número de valvas perpendiculares ao plano de acamamento em porções do offshore transicional não parece representar verdadeiros patches de lingulídeos. É difícil argumentar a favor de algum evento que tenha carreado estas valvas de modo que elas se estabilizaram perpendicularmente ao substrato, não representando posição de vida real. Por isso argumenta-se a favor daqueles lingulídeos perpendiculares como representantes de animais em vida, seja por motivos de correntes que os carregaram para fora de seus patches, com posterior reescavação de seus terriers, seja por representarem animais viventes em populações localmente menos densas (uma vez que o habitat não é o preferencial). Atestando a favor deste argumento estão os lingulídeos infaunais encontrados em posição de vida associados as suas estruturas de escape (ZABINI et al., 2009; ZABINI \& BOSETTI, 2011) em sedimentos finos, e co-ocorrendo também com outros representantes da Província Malvinocáfrica, além daqueles lingulídeos preservados em posição de vida em concreções, e que ocorreram desta forma em $50 \%$ das amostras coletadas, representando sim um local de vida de uma população. Destaca-se que estudos atualistas já atestaram que os lingulídeos infaunais têm mecanismos para evitar a interrupção do fluxo de água em seu interior por efeito de obstrução da cavidade lofoforal por sedimentos finos (EMIG, 1983).

Zabini et al. (2012) estabeleceram uma conexão entre os modos de ocorrência das valvas de lingulídeos infaunais, e outros organismos associados, aos diferentes tratos de sistemas. O trato de sistemas de nível baixo (TSNB) apresenta fósseis fragmentados que representam proximidade à linha de costa; lingulídeos podem ocorrer ali em posição de vida soterrados por eventos de sufocamento proximal. O trato de sistemas transgressivo (TST) apresenta alta diversidade de fósseis (mais que 3 filos) e depósitos de sufocamento em sua porção média. Nas superfícies de transgressão máxima (MFS) animais de carapaça fosfática, como os lingulídeos infaunais, são preservados em concreções. Cordas e rosetas se preservam abaixo do nível de base de ondas de tempestade. Os tratos de sistemas 
de nível alto (TSNA) têm baixa diversidade, com abundância de valvas fragmentadas.

\section{CONSIDERAÇÕES FINAIS}

Desde os tempos de descobertas como as de Clarke (1913) em que a taxonomia, a descrição e levantamentos das faunas fósseis eram os objetivos principais dos estudos, até os dias atuais, em que as interpretações paleoambientais e correlações mundiais são o foco, muito conhecimento foi obtido da análise dos macrofósseis devonianos da Bacia do Paraná.

Apesar do trabalho de Comniskey (2011) ter realizado uma revisão sistemática dos discinídeos para o Devoniano paranaense, as análises continuam em andamento, visto que as coletas em novos pontos são constantes.

A interação ecológica preservada em discinídeos foi a incrustação de briozoarios em Orbiculoidea baini. Através das análises tafonômicas, verificou-se que os discinídeos das espécies Gigadiscina collis, O. bodenbenderi e Rugadiscina sp. possuem preferência por ambientes mais rasos enquanto que Orbiculoidea baini e O. excentrica são registrados tanto em ambientes rasos quanto em profundos.

Em relação aos lingulídeos infaunais, as espécies ainda necessitam de ampla revisão taxonômica, desde que Emig (2003) delimitou o gênero Lingula como não ocorrendo no Paleozoico. As interações ecológicas preservadas nas carapaças de lingulídeos indicam predação ou parasitismo destes organismos, no entanto, mais estudos e amostras são necessários para a determinação correta dos tipos de interação.

A partir das interpretações tafonômicas foi possível estabelecer correlações entre assinaturas tafonômicas e paleoambientes. Assim, lingulídeos infaunais ocorrem preferencialmente em PV nas regiões de foreshore, como nos mares atuais. Já valvas desarticuladas e fragmentadas (concordantes aos planos de acamamento) ocorrem ao longo do shoreface e offshore transicional. Por re-estruturação ambiental, lingulídeos em PV também podem ser encontrados em ambientes de offshore.

\section{AGRADECIMENTOS}

C.Z. agradece ao CNPq pelo auxílio financeiro (140659/2007-2). J.C.C. agradece a Capes pela bolsa de mestrado concedida. E.P.B. agradece ao CNPq (401796/2010-8 e 479774-2011-0).

\section{REFERÊNCIAS BIBLIOGRÁFICAS}

ANELLI, Luis E.; SIMÕES, Marcello G.; LUCIO, M.P. “Bivalves from the Emsian Australocoelia community Ponta Grossa Formation preserved in situ". Anais da Academia Brasileira de Geociências, v. 69, p. 280 281, 1997. 
BAUMILLER, Tomasz K.; LEIGTHON, Lindsey R.; TOMPHSON, David L. Boreholes in Mississippian spiriferide brachiopods and their implications for Paleozoic gastropod drilling. Paleogeog. Paleoclimatol. Paleoecol., v. 147, p. 283 - 289, 1999.

BOSETTI, Elvio P. “Revisão sistemática dos Lingulida (Brachiopoda: Inarticulata) da Formação Ponta Grossa (Devoniano) Estado do Paraná, Brasil”. 1989. In: CONGRESSO BRASILEIRO DE PALEONTOLOGIA, Curitiba. Anais...Curitiba: SBP, 1989. p.73 - 89.

BERGAMASCHI, Sérgio. Análise estratigráfica do Siluro-Devoniano (Formações Furnas e Ponta Grossa) da sub-bacia de Apucarana, Bacia do Paraná, Brasil. 1999. Tese (Doutorado, Instituto de Geociências), Universidade de São Paulo, São Paulo.

CLARKE, John M. Fósseis Devonianos da Bacia do Paraná. Monographias do Serviço Geológico e Mineralógico do Brasil. Rio de Janeiro. f:1-353, pl. 1-27.1913.

COMNISKEY, Jeanninny C; BOSETTI, Elvio P. “Novo Discinídeo do Devoniano da Bacia do Paraná, Estado do Paraná, Brasil". In: REUNIÃO ANUAL DA SOCIEDADE BRASILEIRA DE PALEONTOLOGIA - Paleo 2011. Boletim da Sociedade Brasileira de Paleontologia, 65, SBP, p. 26. 2012.

COMNISKEY, Jeanninny C. Paleontologia dos Discinidae (Brachiopoda: Linguliformea) da Sucessão devoniana da Bacia do Paraná, Estado do Paraná, Brasil: Revisão sistemática, distribuição geográfica e estratigráfica. Dissertação (Mestrado em Gestão do Território) curso de pós-graduação em Geografia, Universidade Estadual de Ponta Grossa, Ponta Grossa, Paraná. 2011

EMIG, Christian C. "Comportment expérimental de Lingula anatina (Brachiopod: Inarticulé) dans divers substrats meubles (Baie de Mutsu, Japon)". Marine Biology, v.75, p. 207 - 217, 1983.

EMIG, Christian C. "Importance du sediment dans la distribuition des Lingules". Lethaia, v. 17, p. 115 -123, 1984.

EMIG, Christian C. “Ecology of the inarticulated brachiopods”. In: KAESLER, R.L. Treatise on Invertebrate Paleontology. Part H - Brachiopoda. n.1. Boulder, Colorado and Lawrence Kansas: Geological Society of America and University of Kansas, 1997. p. 473-495.

EMIG, Christian C. "Proof that Lingula (Brachiopoda) is not a living-fossil, and emended diagnosis of the Family Lingulidae". Carnets de Géologie/ Notebooks on Geology - Letter 2003/01.

GRAHN, Yngve; MAULLER, Paula Mendlowicz.; BERGAMASCHI, Sérgio; BOSETTI, Elvio Pinto. "Palynology and sequence stratigraphy of three Devonian rock units in the Apucarana Sub-basin (Paraná Basin, South Brazil): Additional data and correlation". Review of Paleobotany and Palynology, n. 198, p. $27-44,2013$.

HOFFMEISTER, Allan P.; KOWALEWSKI, Michał; BAUMILLER, Tomasz K.; BAMBACH, Richard. Drilling predation on Permian brachiopodsand bivalves from the Glass Mountains, West Texas. Acta Paleontologica Polonica, n. 49, p. 443 - 454, 2004.

KOWALEWSKI, Michał; FLESSA, Karl W.; MARCOT, Jonathan D. "Predatory scars in the shells of a Recent lingulid brachiopod: Paleontological and ecological implications". Acta Paleontologica Polonica, n.42, p.497 - 532. 1997.

KOZLOWSKI, Roman. "Fósiles Dévoniensis de l' état de Paraná (Brésil)”. Annales de Paleontologie, Paris, v.8, n.3, p.1-19. 1913.

LABARBERA, Michael. "Mechanisms of spatial competition of Discinisca strigata (Inarticulata: Brachiopoda) in the intertidal of Panama". Biol. Bull., n.168, p. 91 - 105, 1985.

LANGE, Frederico W. "Novos Fósseis devonianos do Paraná". Arquivos do Museu Paranaense, n. 3, p. $223-225,1943$.

MERGL, Michel. "Lingulate brachiopods of the Silurian and Devonian of the Barrandian". Acta Musei Nationalis Pragae, Series B - Historia Naturalis, n. 57, n. 1/2, p. 1 - 49, 2001.

MERGL, Michel. "A review of Silurian Discinoid brachiopods from historical British Localities". Bulletin of Geosciences, n.81, 4, 2006. 
MERGL, Michel. “Discinid brachiopod life assemblages: Fossil and extant". Bulletin of Geosciences, n. 85, p. $27-38,2010$.

MERGL, Michel \& MASSA, Dominique. "A new giant discinoid brachiopod from the Lower Devonian of Algeria". Acta Palaeontologica Polonica, n. 2, p. 397-402, 2005.

NUNES, Juliana R.S. Análise tafonômica dos braquiópodes inarticulados (Lingulida) do Membro Jaguariaíva, Formação Ponta Grossa (Devoniano) e suas implicações paleoambientais. 1999. Monografia (Graduação) - Instituto de Biociências, curso de Ciências Biológicas, Universidade Estadual de São Paulo, Botucatu.

PAINE, Robert. "The sediment occupied by Recent lingulid brachiopods and some paleoecological implications". Paleogeog. Paleoclimatol. Paleoecol., n.7, p.21 - 31, 1970.

PETRI, Setembrino; FÚLFARO, V.J. Geologia do Brasil. São Paulo: EDUSP, 1983.

PRITCHARD, David; GLADSTONE, Charlotte. "Reversing bouyancy in turbidity currents: developing a hypothesis fo flow transformation and for deposit facies and architecture". Marine and Petroleum Geology, n. 26, p. 1997 - 2010, 2009.

SCHNEIDER, R.; MUHLMANN, H.; TOMMASI, E.; MEDEIROS, R.A.; DAEMON, R.F.; NOGUEIRA, A.A. "Revisão estratigráfica da Bacia do Paraná. In: CONGRESSO BRASILEIRO DE GEOLOGIA, 28, Porto Alegre. Anais... São Paulo: Sociedade Brasileira de Geologia,1974. p. 41 - 65.

SHARPE, Daniel. "Paleozoic fossils from South Africa”. Quarterly Journal of the Geological Society, p. 210, 1856.

SIMÕES, Marcello G; KOWALEWSKI, Michał; TORELLO, Fernanda F.; GHILARDI, Renato P. “Devonian and Permian benthic marine invertebrates preserved in life position: taphonomic feedback in a Paleozoic epeiric sea". In: CONGRESSO BRASILEIRO DE PALEONTOLOGIA, XVI, Crato. Anais...Crato: Sociedade Brasileira de Paleontologia, 1999a. p. 109 - 110.

SIMÕES, Marcello G.; MARQUES, A.C.; MELLO, L.H.C; GHILARDI, Renato Pirani. “Does the preservation potential play a role in cladistic analyisis?". In: CONGRESSO BRASILEIRO DE PALEONTOLOGIA, XVI, Crato. Anais...Crato: Sociedade Brasileira de Paleontologia, 1999b, p. 109 - 110.

SPARKS, R. Stephen J.; BONNECAZE, Roger T.; HUPPERT, Herbert E.;

LISTER, John R.; HALLWORTH, Mark A.; MADER, Heidy; PHILLIPS, Jeremy. "Sediment-laden gravity currents with reversing buoyancy". Earth and Planetary Science Letters, v.114, p. 243 - 257, 1993

STOW, Dorrik A.V. \& WETZEL, Andreas. "Hemiturbidite: a new type of deep-water sediment". Proceedings of the Ocean Drilling Program, Scientific Results, v.116, p. 25 - 34, 1990.

SÜDKAMP, Wouter H. "Discovered of soft parts of a fossil brachiopod in the "Hunrsücksciefer" (Lower Devonian, Germany)”. Paläontologische Zeitschrift, v. 71, p. 91 - 95, 1997.

WALKER, Roger G.; PLINT, A.Guy. "Wave-and storm-dominated shallow marine systems". Facies Models-response to sea level change. Assoc Canada, p. 219-238, 1992.

WILLIANS, Alwyn; ROWELL, Albert J. In: Moore, ed., Treatise on Invertebrate Paleontology. Part H, Brachiopoda. The Geological Society Of America \& The University of Kansas Press. New York \& Lawrence. v 1, 1997.

WILSON, Mark A. \& PALMER Timothy J. “Domiciles, not predatory borings: a simpler explanation of the holes in Ordovician shells analyzed by Kaplan and Baumiller", 2000. Palaios, v. 16, p. 524 - 525, 2001.

WORCESTER, William S. “On Lingula reevei”. 1969. M.s. Thesis, dept. of Oceanography, Univ. of Hawaii. pp. 49, Havaí.

YATSU, Naohide. “On the habits of the Japonese Lingula”. Annot. Zool. v.4, p. 61 - 67, 1902.

ZABINI, Carolina. Lingulídeos da Sucessão devoniana da Bacia do Paraná, Região dos Campos Gerais, Brasil: revisão de conceitos biológicos-ecológicos e análise tafonômica básica. 2007. Dissertação (Mestrado em Geociências) - Instituto de Geociências, curso de pós-graduação em Geociências, Universidade Federal do Rio Grande do Sul, Porto Alegre. 
ZABINI, Carolina. Integração da tafonomia e estratigrafia de sequências no estudo dos lingulídeos da sucessão devoniana da Bacia do Paraná. 2011. Tese (Doutorado em Geociências) - Instituto de Geociências, curso de pós-graduação em Geociências, Universidade Federal do Rio Grande do Sul, Porto Alegre.

ZABINI, Carolina; BOSETTI, Elvio P. "Paleoambientes revelados: lingulídeos como paleoindicadores em mares paleozoicos". In: CARVALHO, I.S.; SRIVASTAVA, N.K.; STROHSCHOEN, O.; LANA, C.C. Paleontologia: Cenários da vida. Ed. 1. Rio de Janeiro: Interciência, 2011. p. 289-299.

ZABINI, Carolina; BOSETTI, Elvio P.; HOLZ, Michael. Presença de perfurações em lingulídeos (Brachiopoda: Inarticulata) da Sucessão Devoniana da Sub-Bacia de Apucarana, Bacia do Paraná, Brasil. In: ENCONTRO ANUAL DE INICIAÇÃO CIENTÍFICA E VI ENCONTRO DE PESQUISA DA UEPG, XV, Ponta Grossa. Livro de resumos...Ponta Grossa: Editora UEPG, 2006a. 1 CD-ROM.

ZABINI, Carolina; BOSETTI, Elvio Pinto; HOLZ, Michel. Análise de perfurações em Lingulídeos (Brachiopoda: Linguliformea) da Sucessão Devoniana da Sub-Bacia de Apucarana, Bacia do Paraná, Brasil. In: I SEMANA ACADÊMICA DOS ALUNOS DE PÓS-GRADUAÇÃO EM GEOCIÊNCIAS, 1, Porto Alegre. Livro de resumos... Porto Alegre: 2006b, p.141.

ZABINI, Carolina; MATSUMURA, William Mikio K.; BOSETTI, Elvio P. “Estruturas de escape de Lingulida (Famílias Lingulidae e Obolidae) em depósitos de sufocamento". In: XI Reunião da Sociedade Brasileira de Paleontologia Paleo PR/SC, 2009, Curitiba. Resumos da Paleo PR/Sc 2009. Curitiba, 2009. v. 1. p. 29 - 29.

ZABINI, Carolina, BOSETTI, Elvio P.; HORDODISKY, Rodrigo S.; MATSUMURA, Willian Mikio K. "Lingulídeos: revisão dos conceitos morfo-anatômicos, fisiológicos, reprodutivos, paleo-ecológicos e a importância do grupo no Devoniano da região dos Campos Gerais do Paraná, Brasil." Terr@Plural, n. 1, v. 1, p. $115-122,2007$.

ZABINI, Carolina; HOLZ, Michel; BOSETTI, Elvio Pinto; MATSUMURA, William Mikio Kurita; HORODYSKI, Rodrigo Scalise. "Sequence stratigraphy and taphonomic signatures of marine invertebrates: a Devonian (Pragian/Eifelian) example of the Paraná Basin". Journal of South American Earth Sciences, n. 133, p. 8-20. 2012.

ZONNEVELD, John-Paul; GREENE, Sarah E. "Lingulide response to severe storms recorded in middle Triassic strata of northeastern British Columbia". Palaios, n.25, p. 807 - 817, 2010.

Recebido em 23/10/2013

Aceito para publicação em 02/12/2013 